\title{
Utilization of Localized Surface Plasmon Resonance of Silver Nanoparticles for the Spectrofluorimetric Estimation of Oxymetazoline in Dosage Forms: Application to Aqueous Humor
}

Shymaa M. Abd Elhaleem ( $\sim$ Shaimaamostafa154@gmail.com )

Mansoura University https://orcid.org/0000-0003-3816-664X

Fawzi Elsebaei

Mansoura University

Shereen Shalan

Mansoura University

Fathalla Belal

Mansoura University

\section{Research Article}

Keywords: Oxymetazoline, silver nanoparticle, spectrofluorimetry, content uniformity testing, aqueous humor.

Posted Date: July 7th, 2021

DOI: https://doi.org/10.21203/rs.3.rs-551590/v1

License: (c) (1) This work is licensed under a Creative Commons Attribution 4.0 International License.

Read Full License 


\section{Abstract}

A simple, novel, cost-effective and highly sensitive spectrofluorimetric method was developed for estimation of the nasal decongestant oxymetazoline (OMZ) whether per se or in its pharmaceutical preparations using colloidal silver nanoparticles (AgNPs). The method is based on the high catalytic potential activity of AgNPs on the fluorescence intensity of OMZ leading to 12-fold increase in its fluorescene intensity. The response was linear over the range of $\mathbf{2 0 . 0}$ to $\mathbf{7 0 0 . 0} \mathrm{ng} / \mathrm{mL}$ with lower detection limit of $5.0 \mathrm{ng} / \mathrm{mL}$ and limit of quantification of $14.0 \mathrm{ng} / \mathrm{mL}$. The proposed method was applied to the assay of commercial nasal drops, nasal spray and aqueous humor. Interference likely to be encountered from co-administered drugs was studied. The developed method was optimized and validated as per International Council of Harmonization (ICH). An explanation for the drug-AgNPs interaction was proposed.

\section{Introduction:}

Nanoscience includes the study of the materials within nanoscale $(1-100 \mathrm{~nm})$. Among the metal nanoparticles, AgNPs are the most widely used owing to their remarkable chemical and physical properties, including surface enhanced Raman scattering, optical action, electrical conductivity, high thermal, chemical stability and catalytic activity[1]. AgNPs can be used in drug delivery and in the determination of drugs in pharmaceuticals. Although AgNPs have no fluorescence, they were frequently used in the spectrofluorometric analysis of some drugs in biological fluids and dosage forms. They can cause either quenching or enhancement of the fluorescence of drug fluorophores and many drugs were determined based on this fact [2].

Localized surface plasmon resonance (LSPR) is a kind of surface plasmon excitations. It occurs when light hits a nanoparticle having smaller size than the wavelength of the incident light. The incident photon may merge with the metal electrons and start to oscillate coherently[3]. Recent investigations have confirmed that LSPR is an influential technique in biomedical applications due to its sensitivity to material type, size and dielectric constant[4,5], in addition to its impact on enhancing sensitivity of different spectroscopic techniques including fluorescence[6].

Oxymetazoline hydrochloride (OMZ) Fig. 1 [6-tert-butyl-3-(4,5-dihydro-1H-imidazol-2-ylmethyl)-2,4dimethylphenol hydrochloride] [7] is a direct acting synthetic adrenergic agonist that stimulates both $a_{1}$ and $\mathrm{a}_{2}$ adrenergic receptors. Oxymetazoline is found in several nasal spray decongestants and ophthalmic drops. It directly stimulates a receptors on blood vessels supplying the nasal mucosa and conjunctiva, thereby producing vasoconstriction and decreasing congestion[8].It is absorbed from systemic circulation regardless of the route of administration [9] .OMZ is official in each of the United States Pharmacopoeia(USP)[10], the British Pharmacopoeia (BP)[11], and the European Pharmacopoeia [12].Reviewing the literature revealed few reports for $O M Z$ determination ,viz, spectrophotometry[13-18], spectrofluorimetry, ,[19, 20] gas chromatography[21, 22], HPLC [10][23-28], flow injectionchemiluminescence [29, 30],capillary electrophoresis[31], and electrochemistry[32, 33]. 
The reported spectrofluorimetric methods for $\mathrm{OMZ}$ are less sensitive, tedious and need chemical derivatization with either NBD-Cl reagent with linearity range $\left(1-12 \mu \mathrm{g} \mathrm{mL}^{-1}\right)$ [19] or mucin with concentration range from (2.69 to $\left.26.9 \times 10^{-5} \mathrm{~mol} \mathrm{~L}^{-1}\right)$ [20], while the proposed study which is based on its interaction with AgNPs leads to 12-fold increase of its fluorescence and hence, a highly more sensitive method for its determination.

\section{Experimental}

\subsection{Materials and reagents:}

All chemicals used were of Analytical Reagent Grade.

- Authentic sample of oxymetazoline hydrochloride with a purity of $99.9 \%$ was kindly provided by Pharaonia Pharmaceutical Co., Pharo-Pharma, Alexandria, Egypt.

- Oxymet ${ }^{\circledR}$ drops (Batch No. 555 0033) containing $0.5 \mathrm{mg} \mathrm{OMZ/} 1 \mathrm{~mL}$, product of the Pharaonia Pharmaceuticals Co., Pharo-Pharma, Alexandria, Egypt.

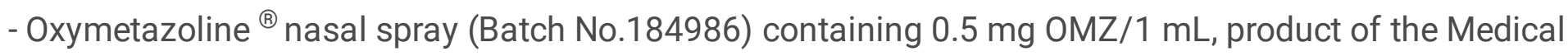
Union Pharmaceuticals Co., Cairo, Egypt.

- Both preparations were obtained from the local market.

- Methanol, acetonitrile, ethanol, boric acid, sodium hydroxide, sodium acetate trihydrate and acetic acid $96 \%$ were obtained from Sigma-Aldrich, Steinheim, Germany.

- Sodium borohydride, silver nitrate and polyvinyl povidone were purchased from Sigma-Aldrich (USA).

\subsection{Instruments:}

- Measurements of fluorescence intensity were done using Cary Eclipse Fluorescence Spectrophotometer equipped with Xenon flash lamp. The method was conducted at smoothing factor of 20, high voltage mode (800 V), and slit width of $5 \mathrm{~nm}$.

- Spectrophotometer: UV/Vis. JENWAY Model 6850.

- pH-meter: Consort, P-901, Belgium.

- Magnetic stirrer: product of Daihan Scientific Co, limited, S. korea.

- Vortex Mixer: (IVM-300p, Taiwan)

- Transmission electron microscope (TEM), JEOL, JSM -2100 (Tokyo, Japan). Sample was loaded on 200 mesh carbon coated Cu-grid and examined at $200 \mathrm{KV}$. 


\subsection{Preparation of standard solutions:}

To prepare standard solution of $\mathrm{OMZ}(100.0 \mathrm{mg} / \mathrm{mL}), 10.0 \mathrm{mg}$ of $\mathrm{OMZ} \mathrm{HCl}$ was dissolved in 100 $\mathrm{mL}$ of ethanol in a volumetric flask. The stock solution was diluted using the same solvent to obtain working solution $(10.0 \mathrm{mg} / \mathrm{mL})$ which was further diluted as appropriate.

\subsection{Preparation of silver nanoparticles:}

Colloidal solution of silver nanoparticles was prepared adopting a previously reported method [34] by adding $10 \mathrm{ml}$ of a precursor $\mathrm{AgNO}_{3}\left(1 \times 10^{-2} \mathrm{M}\right)$ dropwise to $30 \mathrm{ml}$ of freshly prepared $\mathrm{NaBH}_{4}$ solution $\left(2 \times 10^{-2} \mathrm{M}\right)$ with vigorous stirring in an ice bath until light yellow solution was obtained. The obtained solution was further stabilized by addition of $5 \mathrm{ml}$ of PVP $(0.3 \% \mathrm{w} / \mathrm{w})$.

\subsection{Procedures:}

\subsubsection{Construction of Calibration Graph:}

The working solutions were prepared by transferring aliquots from the standard solution covering the concentration range (Table 1) to a set of $10 \mathrm{~mL}$ volumetric flasks followed by $0.1 \mathrm{ml}$ of AgNPs $(6 \mathrm{x}$ $10^{-5} \mathrm{M}$ ) then, the volume was completed to the mark using ethanol. After mixing, measurements of fluorescence intensity were done at $306 \mathrm{~nm}$ (excitation at $281 \mathrm{~nm}$ ) against a reagent blank. The corresponding regression equation was derived and a calibration graph was obtained through a plot between RFI and OMZ concentrations ( $\mathrm{ng} / \mathrm{mL})$.

\subsubsection{Analysis of Pharmaceutical formulations:}

The contents of each of five bottles of the nasal drops and nasal spray were mixed well. Accurately measured volumes from both Oxymet ${ }^{\circledR}$ nasal drops and Oxymetazoline ${ }^{\circledR}$ nasal spray were transferred into $100 \mathrm{~mL}$ volumetric flasks and completed to the volume with ethanol. Serial dilutions were made as appropriate to obtain working solutions within the working range of proposed method (Table 1). The procedure described under " 2.5 .1 "was then followed. The contents of each dosage form were calculated using the corresponding regression equation.

\subsubsection{Procedure for Content Uniformity Testing:}

Assay of 10 0xymet ${ }^{\circledR}$ nasal drops individually was conducted using the proposed method. An accurately measured volume was transferred from each separate preparation into $10 \mathrm{~mL}$ volumetric flasks then completed to the volume with ethanol. Serial dilutions were made to prepare 10 volumetric flasks with a final concentration of $500 \mathrm{ng} / \mathrm{mL}$. The procedure described under "2.5.1" was then applied. The recovery of each preparation was determined using the regression equation and the content uniformity was calculated applying the official USP [35] guidelines.

\subsubsection{Application to aqueous humor:}


An aliquot $(1 \mathrm{~mL})$ of aqueous humor was transferred into a series of $10 \mathrm{~mL}$ volumetric flasks. Aliquots of the working solution of $\mathrm{OMZ}$ within the concentration range and $0.1 \mathrm{~mL}$ of AgNPs were quantitatively added, then vortex mixing for 10 second. Complete to the mark with ethanol, then filter. Proceed as described under "2.5.1".

\section{Results And Discussion:}

AgNPs were prepared using silver nitrate as a precursor, sodium borohydride as a reducing agent and PVP as a stabilizing agent. AgNPs stabilization achieves through electrostatic and steric stabilization. Electrostatic stabilization (e.g., sodium citrate) is caused by the repulsion between particles, caused by the electrical double layer formed by ions adsorbed at the surface of particles and the corresponding counter ions. Steric stabilization (e.g., PVP) is achieved due to the coordination of sterically demanding organic molecules and polymers that act as protective shields on the metallic surface. In this study PVP was chosen as a stabilizer to prevent agglomeration of silver nanoparticles. In addition to, the sensitivity of method was better during using PVP in compare to sodium citrate[36].

Ethanolic solution of oxymetazoline exhibits an emission peak at $306 \mathrm{~nm}$ upon excitation at $281 \mathrm{~nm}$, as demonstrated in (Fig.2).The addition of AgNPs was found to enhance the emission band of the drug by 12-folds (Fig.3).It was previously reported that, the free space absorption conditions of the fluorophores is modified upon adding metal-nanoparticles, and this intensely changes the spectra obtained in the absence of metallic nano surfaces[37]. Figure 4 shows the spectra of increasing concentrations of $\mathrm{OMZ}$ upon addition of fixed volume $(0.1 \mathrm{~mL})$ of $\operatorname{AgNPs}\left(6 \times 10^{-5} \mathrm{M}\right)$ at $306 \mathrm{~nm}$.

\subsection{Characterization of PVP capped AgNPs:}

In order to characterize the prepared colloids, UV-Visible spectrum was recorded for the prepared AgNPs showing intense sharp absorption peak at $385 \mathrm{~nm}$ (Fig.5). In addition, TEM images were used for further confirmation for the prepared AgNPs indicating spherical AgNPs with size range of $142 \mathrm{~nm}$ (Fig.6).

\subsection{Synergistic impact based on LSPR of OMZ:}

Metal induced fluorescence enhancement phenomenon is proposed to be due to interactions of the excited fluorophores with surface plasmon resonances in metals. In this study, the interactions are suggested to be between AgNPs and nitrogen atoms of the imidazoline ring of $\mathrm{OMZ}[5,6]$ leading to remarkable enhancement of OMZ fluorescence intensity, as illustrated in Schem.1.

\subsection{Optimization of experimental parameters:}

The ability of AgNPs to enhance the fluorescence intensity of OMZ was affected by different experimental parameters. These parameters include AgNPs volume, diluting solvents, $\mathrm{pH}$, terbium ion 
effect, incubation time. All these factors were carefully studied in order to optimize the performance of the proposed method.

\subsubsection{Effect of AgNPs volume:}

The effect of AgNPs $\left(6 \times 10^{-5} \mathrm{M}\right)$ solution volume was studied, and the maximum fluorescence intensity was obtained using $0.1 \mathrm{~mL}$, higher volumes led to decreasing the fluorescence intensity (Fig.7).

\subsubsection{Effect of diluting solvents:}

Different diluting solvents including; distilled water, methanol, ethanol and acetonitrile were attempted. The impact of diluting solvents was studied using $0.1 \mathrm{~mL}$ of AgNPs added to OMZ (500 ng $/ \mathrm{mL}$ ). Both acetonitrile and ethanol gave the maximum fluorescence intensity (Fig.8) but ethanol was used as optimum diluting solvent in further experiments because it is more green and eco-friendly [38].

\subsubsection{Effect of pH:}

During this study, two buffer solutions were used including $0.2 \mathrm{M}$ acetate buffer with $\mathrm{pH}$ range 3.0-5.0; and 0.2M phosphate buffer with $\mathrm{pH}$ range 6.5-9.5. The $\mathrm{pH}$ was found to have no considerable impact on the enhancement of the fluorescence intensity.

\subsubsection{Effect of terbium (Thb):}

Terbium was added to the reaction mixture, hopefully, it would enhance the fluorescence intensity of OMZ. The method, known as lanthanide-sensitized luminescence, and is applicable to many aromatic ketones and aldehydes and a variety of biologically important compounds[39]. It was found that, Tb has no effect, and hence it was excluded in this study.

\subsubsection{Effect of incubation time:}

The incubation time was investigated upon addition of $0.1 \mathrm{~mL}$ of AgNPs to OMZ solution. The enhancement in fluorescence intensity was studied at different time intervals from starting one to 30 min. The interaction between $\mathrm{OMZ}$ and AgNPs was found to be completed within $1 \mathrm{~min}$ and the fluorescence intensity was stable for more than $30 \mathrm{~min}$.

\subsection{Validation of the proposed method:}

Evaluation of the validity of the proposed method was done according to ICH Q2R1 recommendations[40], where linearity range, LOQ, LOD, precision, accuracy, sensitivity, robustness, selectivity and specificity, were studied.

\subsubsection{Linearity range:}


The fluorescence intensity of OMZ upon addition of AgNPs increased linearly by increasing drug concentration. Rectilinear range $(20.0-700.0) \mathrm{ng} / \mathrm{ml}$ with perfect correlation coefficient of 0.9999 was obtained. The data of statistical analysis proving the linearity of the proposed method are summarized in Table 1

Both LOQ and LOD were determined according to ICH Q2R1 recommendations [40], where the values of LOQ and LOD are $14.0 \mathrm{ng} / \mathrm{mL}$ and $5.0 \mathrm{ng} / \mathrm{mL}$, respectively(Table 1).

\subsubsection{Accuracy and precision:}

The accuracy of the proposed method was attained during comparing the outcomes of the analysis of OMZ samples with the results obtained upon using official USP method (HPLC method) [10] for both raw material and commercial dosage forms.

No considerable differences were observed concerning the accuracy and precision between the performance of the proposed and official USP methods using both Student t-test and variance ratio F-test [41] as illustrated in Table 2.

The precision of the proposed method is investigated through both intra-day and inter-day precisions.

The intra-day precision is tested through measuring three different concentrations of $\mathrm{OMZ}$ and every concentration was repeated three times. While measuring the same concentrations of $\mathrm{OMZ}$ on three consecutive days represented the inter-day precision. The precision of the method is confirmed through the low values of \%RSD which (less than $2 \%$ ) indicating good repeatability and reproducibility (Table 3 ).

\subsubsection{Robustness:}

The robustness was evaluated through testing the impact of minor changes influencing the fluorescence intensity during routine work. The impact of AgNPs volume $(0.1 \mathrm{~mL} \pm 0.02)$ has been studied with no significant effects on recovery percentages and\% RSD values, (Table 4).

\subsubsection{Selectivity:}

\section{Interference study:}

The interference likely to be encountered from some commonly co-administrated and/or coformulated drugs was studied. The tolerance limits of these compounds, namely; Xylometazoline hydrochloride, triamcinolone acetonide and cromolyn sodium, were determined. The high values of the obtained tolerance limits reveal the high selectivity of the proposed method. (Table 5).

\subsection{Applications:}

\subsubsection{Analysis of $O M Z$ in its dosage forms:}


The proposed method was successfully applied to estimate OMZ in its nasal drops and nasal spray preparations. The high percentage recoveries obtained pointed out that no interference was encountered from the existing excipients. A perfect harmonization is achieved between the outcomes recorded in (Table 6) with those found via official USP method [10]. The results of statistical analysis obtained using both Student's t-test and variance ratio F-test [38] indicated no considerable differences between the proposed method and the USP method performance.

\subsubsection{Content uniformity testing:}

Due to remarkable sensitivity of the proposed method, it was exploited for content uniformity testing. The fluorescence intensity of Oxymet ${ }^{\circledR}$ nasal drops was determined according to USP guidelines [35]. [39]Dosage uniformity requirements are met as the acceptance value (AV) of the randomly selected 10 preparations is less than to $\mathrm{L} 1(\mathrm{~L} 1=15 \%)$, as illustrated in (Table 7 ).

\subsubsection{Analysis of $O M Z$ in aqueous humor:}

The high sensitivity of the proposed method allowed the determination of OMZ in aqueous humor. The results revealed that the mean absolute recoveries and the SD of OMZ in aqueous humor is $100.05 \pm$ 1.06 (Table 8).

\section{Conclusion:}

AgNPs are one of metal nanoparticles, that are chemically synthesized using reducing agent (sodium borohydride), stabilizing agent (PVP) and a precursor $\left(\mathrm{AgNO}_{3}\right)$. The PVP coated AgNPs caused significant enhancement of $\mathrm{OMZ}$ fluorescence. There is no encountered interference from other drugs and ingredients during determination of $\mathrm{OMZ}$ in dosage forms or aqueous humor.

\section{Declarations}

\section{Funding.}

No funding was received for conducting this study.

\section{Conflict of Interest}

The authors declare that they have no conflict of interest.

\section{Availability of data and materials}

All the data and the materials are available all-over the study.

\section{Code Availability}

Not applicable. 
CRediT authorship contribution statement

Shymaa M. Abd Elhaleem: Methodology, Formal analysis, Validation, Investigation, Writing - original draft.

Fawzi Elsebaei: Validation, Writing - Review \& Editing, Supervision.

Shereen Shalan: Validation, Writing - Review \& Editing, Supervision.

Fathalla Belal: Conceptualization, Validation, Writing - Review \& Editing, Resources, Supervision.

\section{References}

[1] K. Alaqad, T.A. Saleh, Gold and silver nanoparticles: synthesis methods, characterization routes and applications towards drugs, J. Environ. Anal. Toxicol., 6 (2016) 525-2161.

[2] A. Jouyban, E. Rahimpour, Optical sensors based on silver nanoparticles for determination of pharmaceuticals: An overview of advances in the last decade, Talanta, (2020) 121071.

[3] K.A. Willets, R.P. Van Duyne, Localized surface plasmon resonance spectroscopy and sensing, Annu. Rev. Phys. Chem., 58 (2007) 267-297.

[4] J. Khodaveisi, A.M.H. Shabani, S. Dadfarnia, M.R. Moghadam, M.R. Hormozi-Nezhad, Simultaneous determination of protocatechuic aldehyde and protocatechuic acid using the localized surface plasmon resonance peak of silver nanoparticles and chemometric methods, Quím. Nova, 38 (2015) 896-901.

[5] M.R. El-Zahry, A localized surface plasmon resonance sensing method for simultaneous determination of atenolol and amiloride in pharmaceutical dosage forms and urine samples, J. Anal. Methods Chem., 2018.

[6] N. Alarfaj, S. Altamimi, M. El-Tohamy, A. Almahri, Exploitation of localized surface plasmon resonance of silver/gold nanoparticles for the fluorescence quantification of angiotensin II receptor antagonists in their tablets and bio-samples, New J. Chem., 43 (2019) 492-503.

[7] Moffat, A.C., Osselton, M.D., Widdop, B., Galichet, L.Y.; Clark's analysis of drugs and poisons in pharmaceuticals, body fluids and postmortem material; Vol. (II), 4th Ed. The Pharmaceutical Press, London, (2011): 1845

[8] Sweetman, S. Martindale; The complete drug reference. the Pharmaceutical Press, London,38 ed,2014; Vol. A,P.1671.

[9] K. Whalen, Lippincott illustrated reviews: pharmacology, Lippincott Williams \& Wilkins, Philadelphia, 2018. 
[10] The United States Pharmacopoeia 34, the National Formulary 29; the US Pharmacopoeial Convention: Rockville, MD, (2011), Vol. III,P. 3782

[11] The British Pharmacopoeia, Her Magesty's Stationary Office: London, 2019; Vol. II, p. 499.

[12] The European Pharmacopoeia 7: Council Of Europe : European Directorate for the Quality of Medicines and Healthcare, Strasbourg, 2011; Vol. (II),P.2648.

[13] T.a.N. Al-Sabha, B.A. Rasheed, Spectrophotometric determination of oxymetazoline hydrochloride based on the oxidation reactions, Jordan J. Chem., 146 (2011) 1-9.

[14] S.A. Zakaria, Spectrophotometric determination of oxymetazoline hydrochloride via oxidative coupling reaction with 4-aminoantipyrine in the presence of potassium periodate, Rafidain J. Sci., 22 (2011) 97-108.

[15] O. Abdel-Aziz, A. El-Kosasy, N. Magdy, N. El Zahar, Novel spectroscopic methods for determination of Cromolyn sodium and Oxymetazoline hydrochloride in binary mixture, Spectrochim. Acta A, 131 (2014) 59-66.

[16] M.A.M. Hegazy, M.A. Al-Ghobashy, B.M. Eltanany, F.I. Khattab, Spectral resolution and simultaneous determination of oxymetazoline hydrochloride and sodium cromoglycate by derivative and ratio-based spectrophotometric methods, Eur. J. Chem., 6 (2015) 319-324.

[17] N.S. Othman, S.A. Fathe, Indirect spectrophotometric determination of oxymetazoline hydrochloride, Rafidain J.Sci., 24 (2013) 84-95.

[18] J.O. Abdulsattar, H. Hadi, S. Richardson, A. Iles, N. Pamme, Detection of doxycycline hyclate and oxymetazoline hydrochloride in pharmaceutical preparations via spectrophotometry and microfluidic paper-based analytical device ( $\mu$ PADs), Anal. Chim. Acta, 1136 (2020) 196-204.

[19] F. Salama, K. Abdel-Salam, A. Abdel-Halim, R. Said, A. Madkour, Validated Spectrofluorimetric Method for The Determination of Oxymetazoline Hydrochloride via Derivatization with 4-Chloro-7-nitrobenzo-2oxa-1, 3-diazole (NBD-Cl), Eurasian J. Anal. Chem., 13 (2018) 4.

[20] X. Yu, H. Liu, Y. Yang, S. Lu, Q. Yao, P. Yi, The investigation of the interaction between Oxymetazoline hydrochloride and mucin by spectroscopic approaches, Spectrochim. Acta A, 103 (2013) 125-129.

[21] P. Boon, W. Sudds, The gas chromatographic determination of imidazolines in pharmaceutical preparations, J. Pharm. Pharmacol., 19 (1967) Suppl: 88S.

[22] M. Massaccesi, Gas chromatographic determination of some imidazolines in pharmaceutical preparations using the FFAP in stationary phase, Pharm. Acta Helv., 62 (1987) 302. 
[23] T. Hoffmann, R. Thompson, J. Seifert, Determination of the nasal decongestant, oxymetazoline hydrochloride, in pharmaceutical formulations by HPLC, Drug Dev. Ind. Pharm., 15 (1989) 743-757.

[24] B. Stanisz, W. Nowinski, Determination of oxymetazoline hydrochloride and decomposition products by high-performance liquid chromatography, Acta Pol. Pharm., 57 (2000) 399-402.

[25] S. Sudsakorn, L. Kaplan, D.A. Williams, Simultaneous determination of triamcinolone acetonide and oxymetazoline hydrochloride in nasal spray formulations by HPLC, J. Pharm. Biomed. Anal., 40 (2006) 1273-1280.

[26] K.A. Shaikh, A.T. Patil, Stability-indicating HPLC method for the determination of mometazone furoate, oxymetazoline, phenyl ethanol and benzalkonium chloride in nasal spray solution, J. Trace Anal., Food \&Drugs, 1 (2013) 14-21.

[27] M.A. Hegazy, M.A. Al-Ghobashy, B.M. Eltanany, F.I. Khattab, Validated chromatographic methods for the simultaneous determination of sodium cromoglycate and oxymetazoline hydrochloride in a combined dosage form, J. Advances Chem., 11 (2015).

[28] M. Fathy, S. Abo El Abass Mohamed, H. Elmansi, F. Belal, Simultaneous Determination of Cromolyn Sodium Combined Dosage Forms Using Isocratic HPLC Method, J. Chromatogr. Sci., 55 (2016) 14-22.

[29] A.M. García-Campaña, J.M.B. Sendra, M.P.B. Vargas, W.R. Baeyens, X. Zhang, Flow injection analysis of oxymetazoline hydrochloride with inhibited chemiluminescent detection, Anal. Chim. Acta, 516 (2004) 245-249.

[30] N.N. Wang, Y.Q. Shao, Y.H. Tang, H.P. Yin, X.Z. Wu, Flow-injection chemiluminescence method for the determination of naphazoline hydrochloride and oxymetazoline hydrochloride, J. Lumin., 24 (2009) 178182.

[31] Q. Chen, P. Li, H. Yang, B. Li, J. Zhu, L. Peng, Nonaqueous capillary electrophoresis conditions for the simultaneous separation of eight alpha-adrenergic blocking agents, Anal. Bioanal. Chem., 398 (2010) 937-942.

[32] A.M. Abdel-Raoof, A.H. Abdel-Monem, A.A. Almrasy, T.F. Mohamed, Z.A. Nasr, G.F. Mohamed, Optimization of Highly Sensitive Screen Printed Electrode Modified With Cerium (IV) Oxide Nanoparticles for Electrochemical Determination of Oxymetazoline Hydrochloride Using Response Surface Methodology, J. Electrochem. Soc., 167 (2020) 047502.

[33] A. Munir, B. Bozal-Palabiyik, A. Khan, A. Shah, B. Uslu, A novel electrochemical method for the detection of oxymetazoline drug based on MWCNTs and TiO2 nanoparticles, J. Electroanal. Chem., 844 (2019) 58-65.

[34] K. Mavani, M. Shah, Synthesis of silver nanoparticles by using sodium borohydride as a reducing agent, Int. J. Eng. Res. Technol., 2 (2013) 1-5. 
[35] J.M. Martins, A. Farinha, Uniformity of dosage units-comparative study of methods and specifications between Eur. Pharm. 3rd and USP 23, J. Pharm. Biomed., 18 (1998) 487-495.

[36] J. Tashkhourian, M. Hormozi-Nezhad, J. Khodaveisi, Application of silver nanoparticles and principal component-artificial neural network models for simultaneous determination of levodopa and benserazide hydrochloride by a kinetic spectrophotometric method, Spectrochim. Acta A, 82 (2011) 25-30.

[37] J. Leiterer, F. Delissen, F. Emmerling, A. Thünemann, U. Panne, Structure analysis using acoustically levitated droplets, Anal. Bioanal. Chem., 391 (2008) 1221-1228.

[38] M. Tobiszewski, J. Namieśnik, Greener organic solvents in analytical chemistry, Curr. Opin. Green Sustain., 5 (2017) 1-4.

[39] J. Georges, Lanthanide-sensitized luminescence and applications to the determination of organic analytes. A review, Analyst, 118 (1993) 1481-1486.

[40] I.S. Committee, ICH harmonised tripartite guidelines. Validation of analytical procedures: methodology Q2, in: International Conference on Harmonisation of Technical Requirements for Registration of Pharmaceuticals for Human Use. Step, 1996.

[41] J. Miller, J.C. Miller, Statistics and chemometrics for analytical chemistry, Pearson education, 2018.

\section{Tables}

Due to technical limitations, table 1-8 is only available as a download in the Supplemental Files section.

\section{Figures}




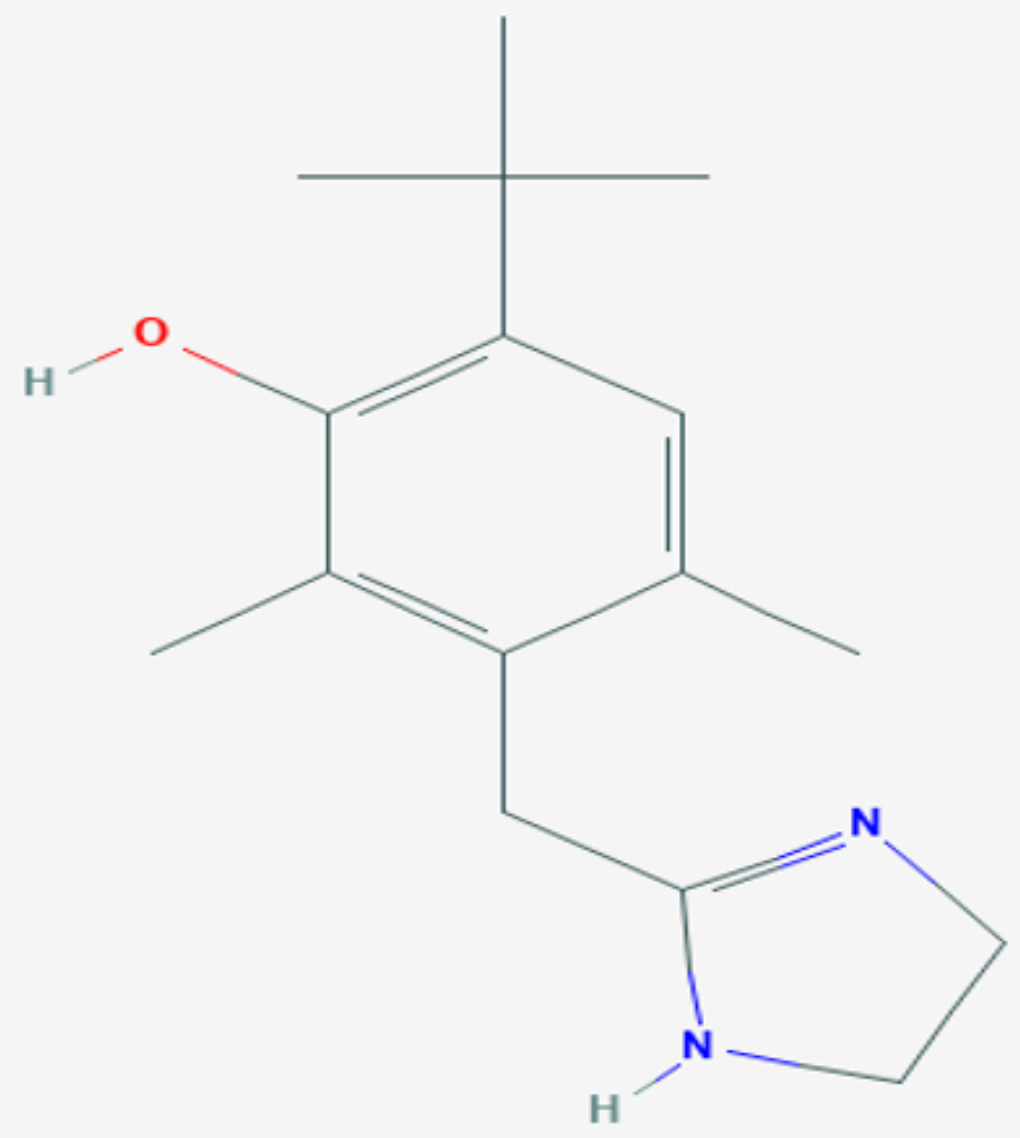

$\mathrm{Cl}-\mathrm{H}$

Fig.1

Figure 1

Chemical structure of oxymetazoline hydrochloride. 


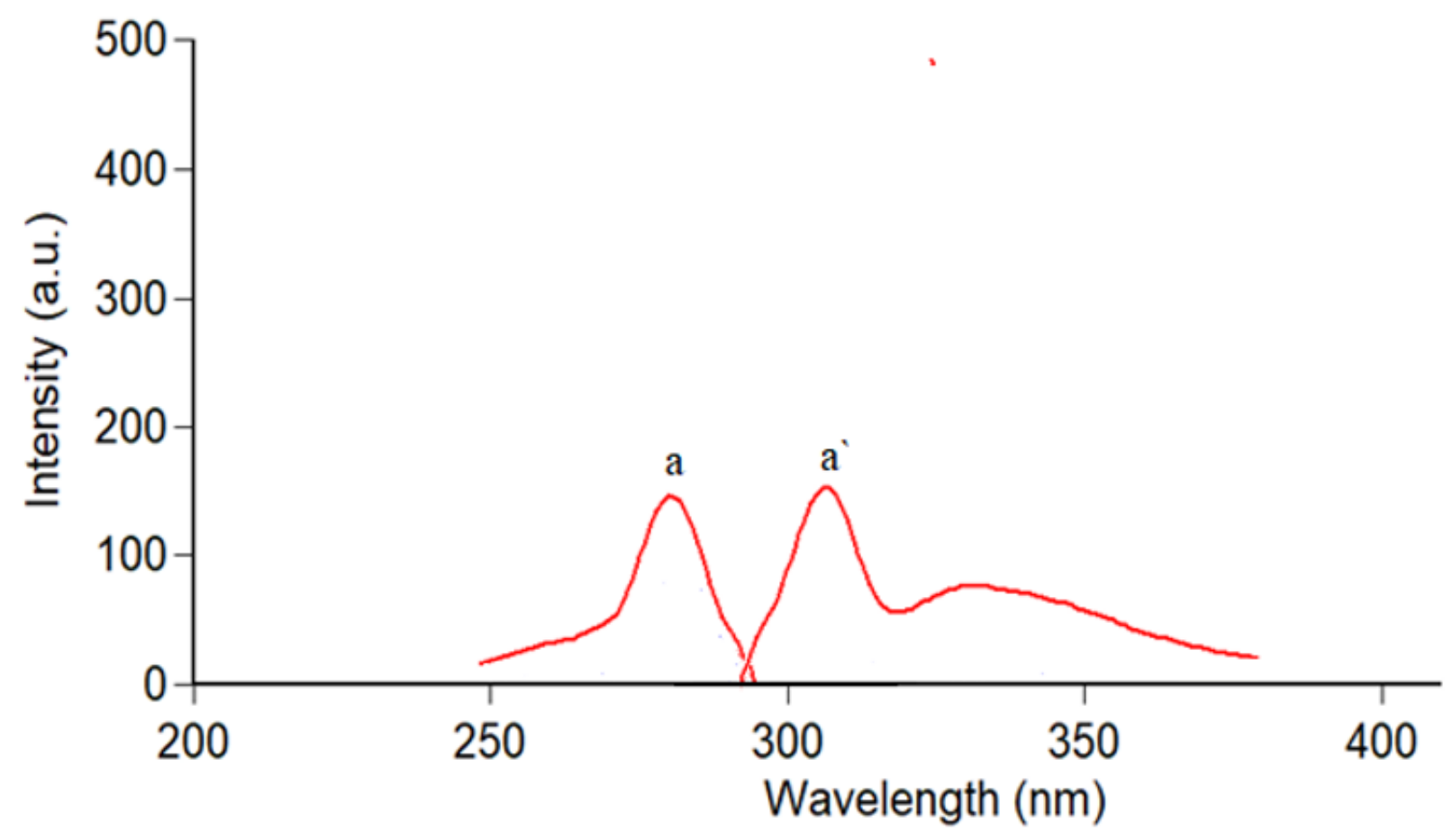

Fig.2

\section{Figure 2}

Excitation and emission spectra of $\mathrm{OMZ}(500 \mathrm{ng} / \mathrm{mL})$ in ethanol(a,a').

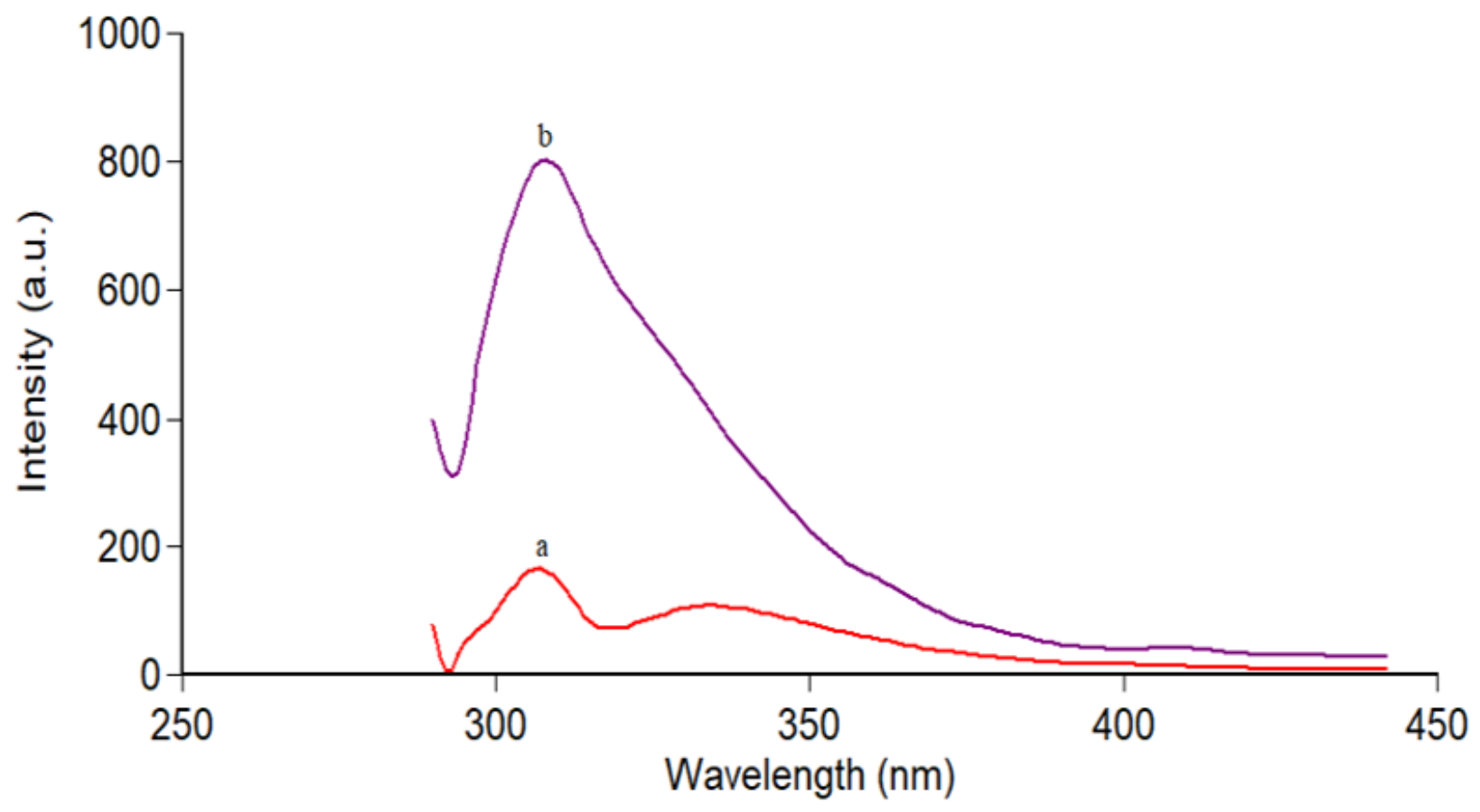

Fig.3 
Figure 3

Emission spectra of OMZ in ethanol. Where: (a): OMZ emission peak at concentration $(500 \mathrm{ng} / \mathrm{mL})$. (b): $\mathrm{OMZ}$ - AgNPs emission peak when the concentration of OMZ is $(500 \mathrm{ng} / \mathrm{mL})$ using $0.1 \mathrm{~mL}$ of AgNPs $(6 \mathrm{x}$ $10-5 \mathrm{M})$.

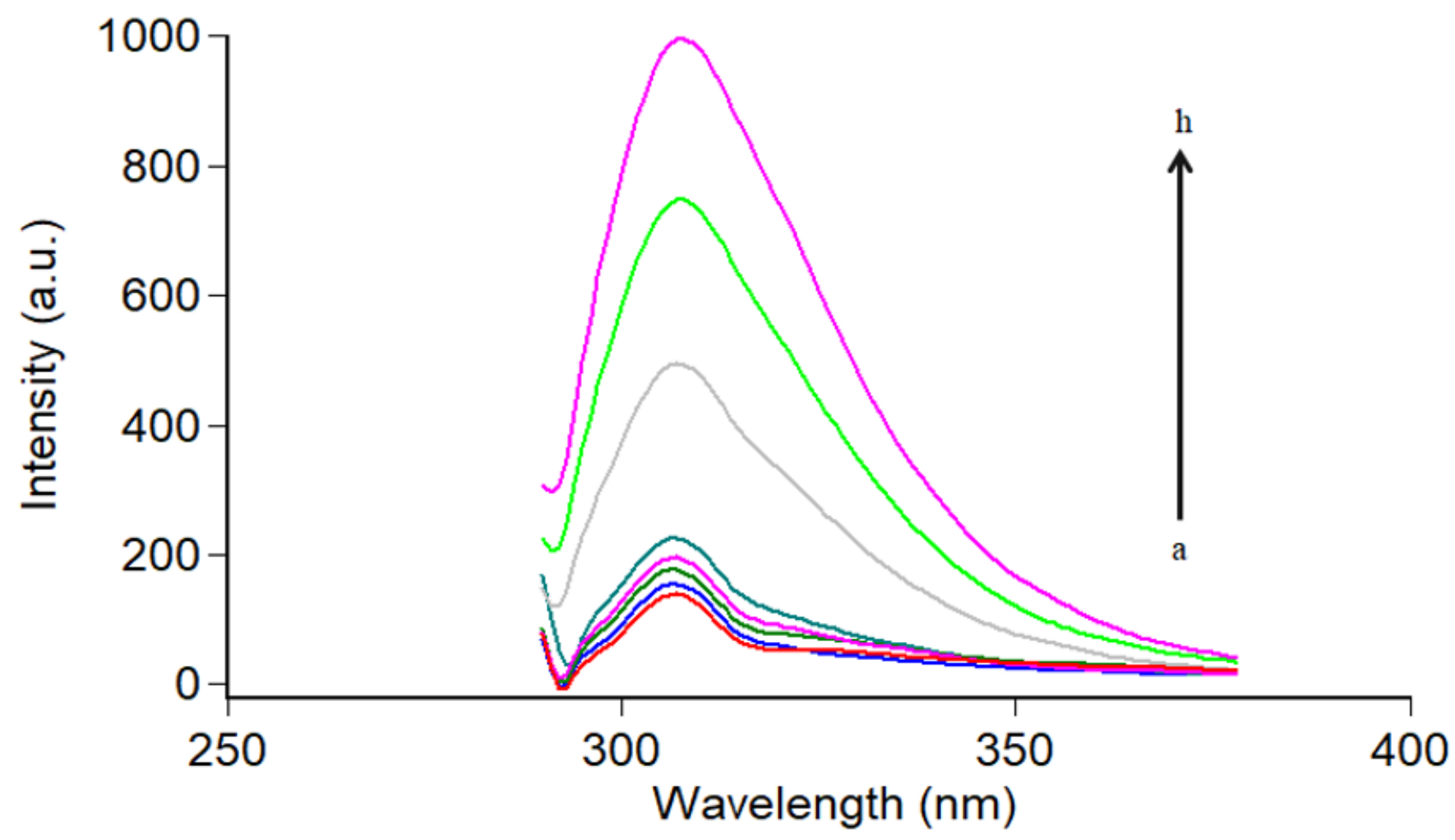

Fig.4

\section{Figure 4}

Emission spectra for increasing concentrations of $\mathrm{OMZ}$ in ethanol during using $0.1 \mathrm{~mL}$ of AgNPs $(6 \times 10$ $-5 \mathrm{M})$, where (a-h) are (20.0,30.0, 50.0,70.0, 100.0, 300.0, 500.0, 700.0 ng/mL OMZ). 


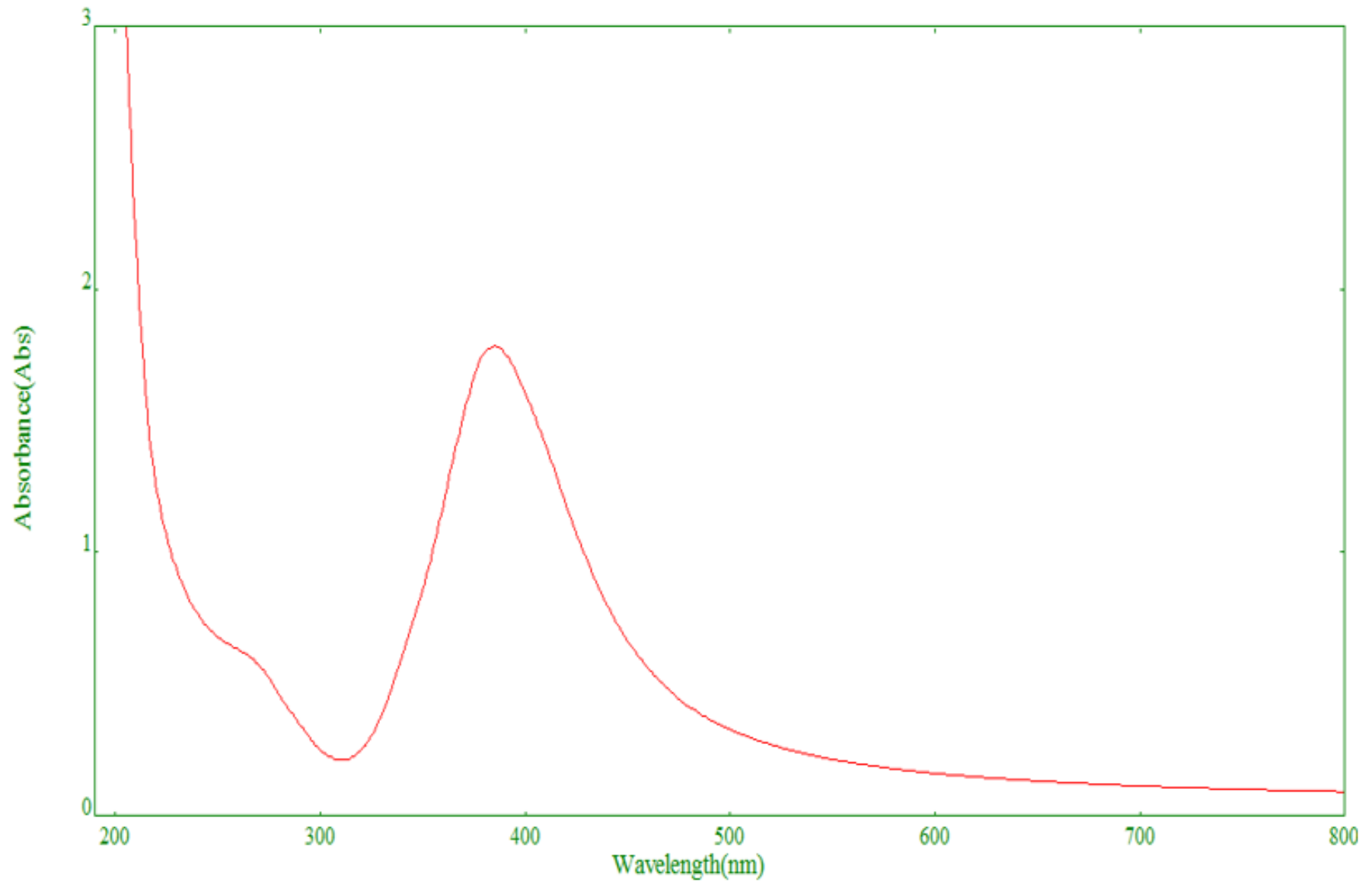

Fig.5

Figure 5

UV-visible spectrum of the prepared AgNPs ( 6 × $10-5 \mathrm{M})$. 

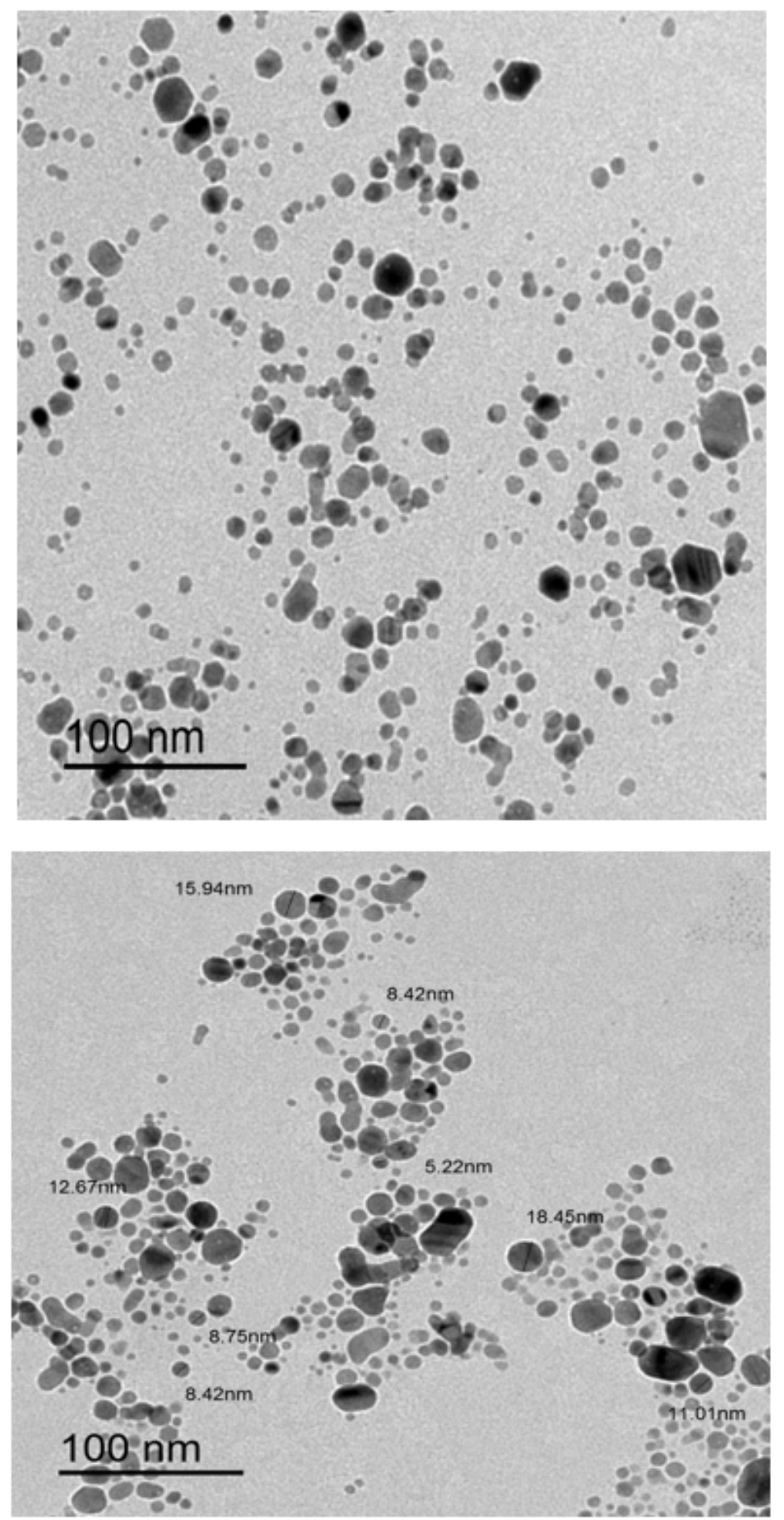

Fig.6

Figure 6

TEM images of the prepared AgNPs $(6 \times 10-5 \mathrm{M})$. 


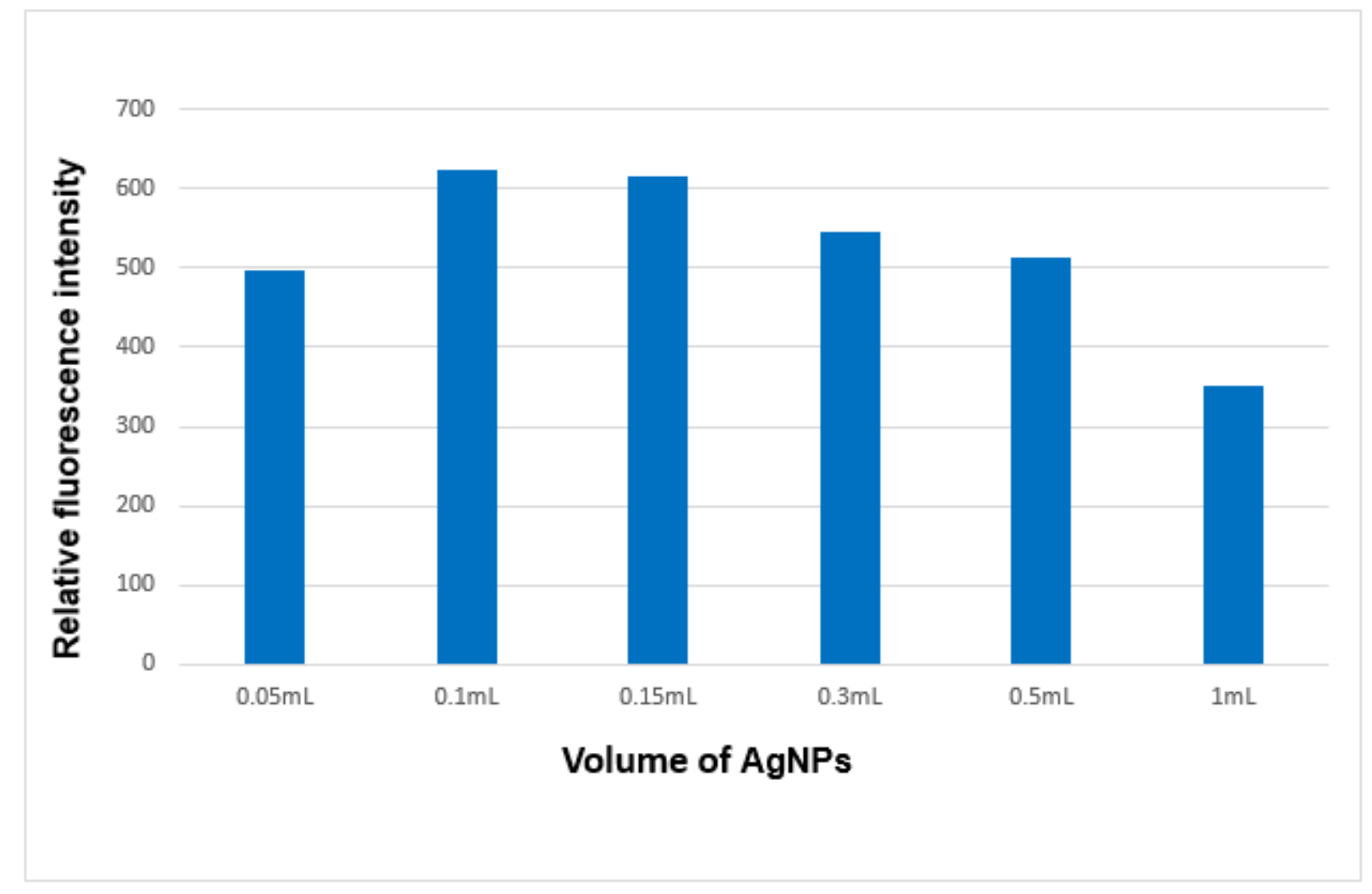

Fig.7

Figure 7

Effect of volume of AgNPs ( $6 \times 10-5 \mathrm{M})$ on fluorescence intensity of OMZ (500 ng/mL). 


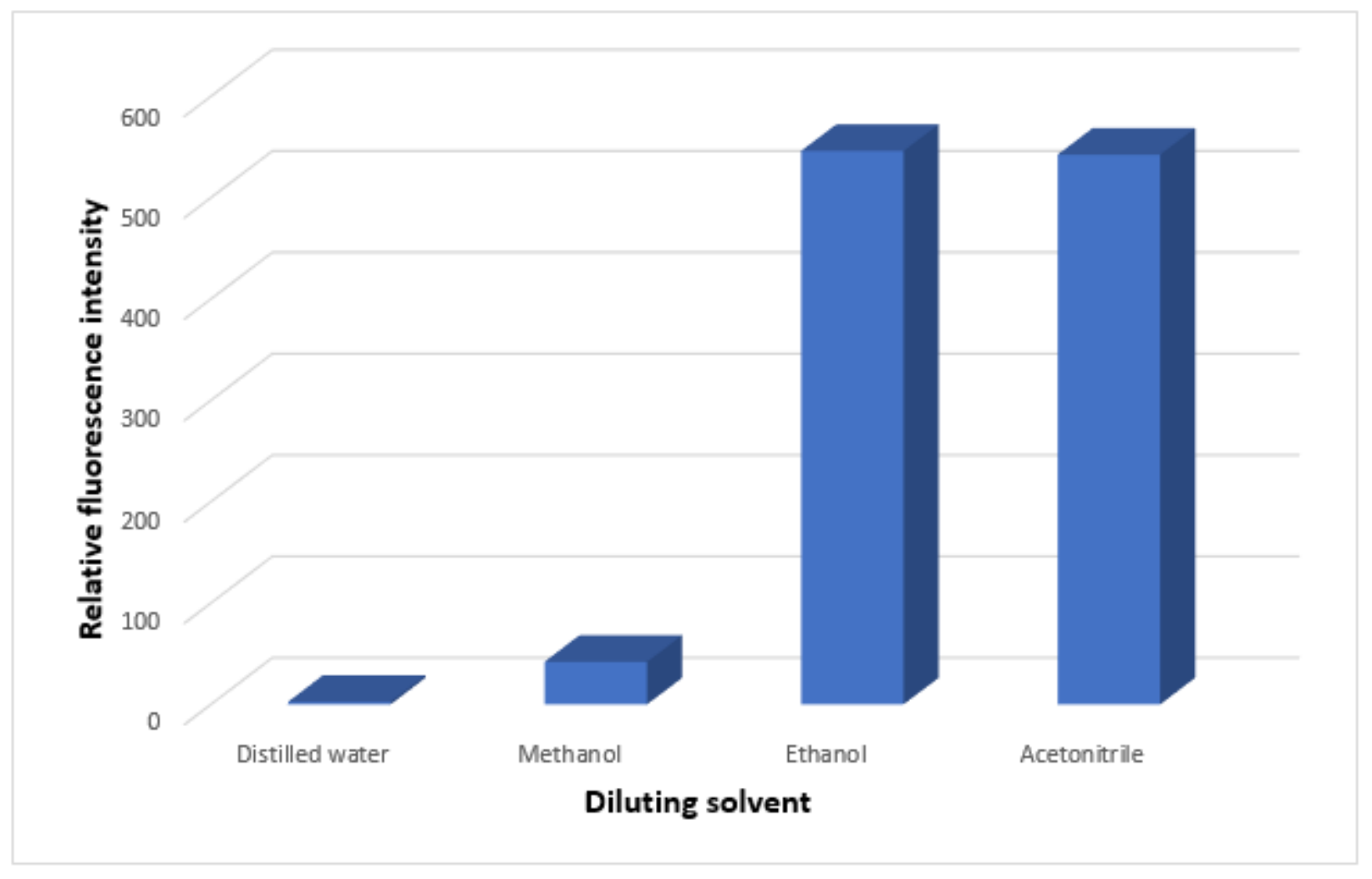

Fig.8

Figure 8

Effect of diluting solvents on the fluorescence intensity of OMZ $(500 \mathrm{ng} / \mathrm{mL})$ using $0.1 \mathrm{~mL}$ AgNPs $(6 \times 10$ $-5 \mathrm{M})$.

\section{Supplementary Files}

This is a list of supplementary files associated with this preprint. Click to download.

- Tables.pdf

- Schem1.docx 